\title{
Prediction Model for Tumor Budding Status Using the Radiomic Features of F-18 Fluorodeoxyglucose Positron Emission Tomography/Computed Tomography in Cervical Cancer
}

\author{
Gun Oh Chong $1,2,3,+\infty$, Shin-Hyung Park ${ }^{4,5,+}$, Shin Young Jeong ${ }^{6,7, *}$, Su Jeong Kim ${ }^{1,2}$, Nora Jee-Young Park ${ }^{3,8} \mathbb{C}^{\mathbb{D}}$, \\ Yoon Hee Lee ${ }^{1,2,3}$, Sang-Woo Lee ${ }^{6,7}$, Dae Gy Hong ${ }^{1,2}$, Ji Young Park ${ }^{8}$ (i) and Hyung Soo Han ${ }^{3,9}$ (D) \\ 1 Department of Obstetrics and Gynecology, School of Medicine, Kyungpook National University, \\ Daegu 41944, Korea; gochong@knu.ac.kr (G.O.C.); sujeong1129@naver.com (S.J.K.); \\ mylyh@naver.com (Y.H.L.); chssa0220@hanmail.net (D.G.H.) \\ 2 Department of Obstetrics and Gynecology, Chilgok Hospital, Kyungpook National University, \\ Daegu 41404, Korea \\ 3 Clinical Omics Research Center, School of Medicine, Kyungpook National University, Daegu 41944, Korea; \\ pathpjy@naver.com (N.J.-Y.P.); hshan@knu.ac.kr (H.S.H.) \\ 4 Department of Radiation Oncology, School of Medicine, Kyungpook National University, \\ Daegu 41944, Korea; shinhyungpark@knu.ac.kr \\ check for \\ updates \\ Citation: Chong, G.O.; Park, S.-H.; \\ Jeong, S.Y.; Kim, S.J.; Park, N.J.-Y.; \\ Lee, Y.H.; Lee, S.-W.; Hong, D.G.; \\ Park, J.Y.; Han, H.S. Prediction Model \\ for Tumor Budding Status Using the \\ Radiomic Features of F-18 \\ Fluorodeoxyglucose Positron \\ 5 Cardiovascular Research Institute, School of Medicine, Kyungpook National University, Daegu 41944, Korea \\ 6 Department of Nuclear Medicine, School of Medicine, Kyungpook National University, Daegu 41944, Korea; \\ swleenm@knu.ac.kr \\ 7 Department of Nuclear Medicine, Chilgok Hospital, Kyungpook National University Daegu, \\ Daegu 41404, Korea \\ 8 Department of Pathology, School of Medicine, Kyungpook National University, Daegu 41944, Korea; \\ jyparkmd@knu.ac.kr \\ 9 Department of Physiology, School of Medicine, Kyungpook National University, Daegu 41944, Korea \\ * Correspondence: syjeong@knu.ac.kr; Tel.: +82-53-200-2852; Fax: +82-53-200-2029 \\ + Authors contributed equally to this work as co-first authors.
} Emission Tomography/Computed Tomography in Cervical Cancer. Diagnostics 2021, 11, 1517. https:// doi.org/10.3390/diagnostics11081517

Academic Editors: Angela Santoro and Gian Franco Zannoni

Received: 13 May 2021

Accepted: 18 August 2021

Published: 23 August 2021

Publisher's Note: MDPI stays neutral with regard to jurisdictional claims in published maps and institutional affiliations.

Copyright: () 2021 by the authors. Licensee MDPI, Basel, Switzerland. This article is an open access article distributed under the terms and conditions of the Creative Commons Attribution (CC BY) license (https:// creativecommons.org/licenses/by/ $4.0 /)$.
Abstract: Objective: To compare the radiomic features of F-18 fluorodeoxyglucose positron emission tomography/computed tomography $\left({ }^{18} \mathrm{~F}\right.$-FDG PET/CT) and intratumoral heterogeneity according to tumor budding (TB) status and to develop a prediction model for the TB status using the radiomic feature of ${ }^{18}$ F-FDG PET/CT in patients with cervical cancer. Materials and Methods: Seventy-six patients with cervical cancer who underwent radical hysterectomy and preoperative ${ }^{18} \mathrm{~F}-\mathrm{FDG}$ PET/CT were included. We assessed the status of intratumoral budding (ITP) and peritumoral budding (PTB) in all available hematoxylin and eosin-stained specimens. Three conventional metabolic parameters and fifty-nine features were extracted and analyzed. Univariate analysis was used to identify significant metabolic parameters and radiomic findings for TB status. The prediction model for TB status was built using 3 machine learning classifiers (random forest, support vector machine, and neural network). Results: Univariate analysis led to the identification of 2 significant metabolic parameters and 12 significant radiomic features according to intratumoral budding (ITB) status. Among these parameters, following multivariate analysis for the ITB status, only compacity remained significant (odds ratio, 5.0047; 95\% confidence interval, 1.1636-21.5253; $p=0.0305$ ). Two conventional metabolic parameters and 25 radiomic features were selected by the Lasso regularization, and the prediction model for the ITB status had a mean area under the curve of 0.762 in the test dataset. Conclusion: Radiomic features of ${ }^{18}$ F-FDG PET/CT were associated with the ITB status. The prediction model using radiomic features successfully predicted the TB status in patients with cervical cancer. The prediction models for the ITB status may contribute to personalized medicine in the management of patients with cervical cancer.

Keywords: cervical cancer; tumor budding; radiomic features; ${ }^{18}$ F-FDG PET/CT; prediction model 


\section{Introduction}

Tumor budding (TB) is defined as a single neoplastic cell or cell cluster of up to four neoplastic cells at the invasive front of the tumor (peritumoral budding (PTB)) or within the tumor mass (intratumoral budding (ITB)) [1]. Several studies have demonstrated that TB is associated with lymphovascular invasion (LVI), lymph node metastasis, disease recurrence, and an unfavorable survival outcome, especially in colorectal cancer [2], esophageal carcinoma [3], and head and neck cancer [4]. Recently, we evaluated the prognostic roles of $\mathrm{TB}$ and the correlation between TB and conventional pathological parameters in gynecological cancers $[5,6]$. Our results demonstrated that TB was associated with deep depth invasion, higher International Federation of Gynecologic Obstetrics (FIGO) stage, LVI, and lymph node metastasis in endometrial cancer [5]. Moreover, high TB was an independent prognostic factor for predicting survival outcomes in cervical cancer [6].

Currently, F-18 fluorodeoxyglucose positron emission tomography/computed tomography $\left({ }^{18} \mathrm{~F}\right.$-FDG PET/CT) is widely used to detect lymph node involvement, distant metastasis, and recurrence in cervical cancer [7]. Various metabolic parameters of ${ }^{18} \mathrm{~F}$-FDG $\mathrm{PET} / \mathrm{CT}$ have been reported as prognostic factors, including the maximum standardized uptake value (SUVmax), metabolic tumor volume (MTV), and total lesion glycolysis (TLG). A recent systematic review showed that a high primary tumor SUVmax has a significant correlation with the poor event-free survival (hazard ratio (HR), 1.938; 95\% confidence interval (CI), 1.203-3.054, $p=0.004$ ) and overall survival (HR, 2.582; 95\% CI, 1.936-3.443, $p<0.001$ ). Moreover, the highest primary tumor TLG (HR, 1.843; 95\% CI, 1.100-3.086, $p=0.02)$ and MTV (HR, 2.06; 95\% CI, 1.21-3.51, $p=0.007)$ was associated with poor eventfree survival [8]. Recently, radiomics studies, which represent intratumoral heterogeneity, have emerged as a new and exciting area of research. The measurement of texture indices from tumor ${ }^{18} \mathrm{~F}-\mathrm{FDG}$ PET/CT images has recently been proposed as an adjunct to predict tumor response to therapy. Moreover, there is emerging evidence that intratumoral metabolic heterogeneity on pretreatment ${ }^{18}$ F-FDG PET/CT might be a predictor of tumor recurrence after treatment in patients with lung, esophageal, head and neck, and cervical cancer [9-12]. Furthermore, recent studies have shown that ${ }^{18}$ F-FDG PET/CT radiomics using various textural features are potential biomarkers to predict tumor recurrence and lymph node metastasis [13,14].

Tumor heterogeneity is defined as the presence of different cell subpopulations or clones and has a fundamental role in tumor growth, progression, and therapeutic resistance. Tumor hypoxia, angiogenesis, necrosis, fibrosis, cell proliferation, and inflammation are all known to affect tumor heterogeneity [15]. Epithelial-mesenchymal transition (EMT) of primary tumor tissues may lead to the loss of cell-to-cell adhesion, allowing individual cells or small groups of cells to acquire the ability to migrate and invade through the surrounding tissues. Moreover, TB may lead to more aggressive clinicopathologic characteristics through a similar mechanism of EMT, such as increased extracellular matrix degradation, increased migration, and loss of cell adhesion [16]. These EMT processes are accompanied by changes in cell morphology [17] and may lead to changes in tumor heterogeneity. However, to our knowledge, no previous studies have evaluated the correlation between metabolic parameters and radiomic findings of ${ }^{18} \mathrm{~F}$ - FDG PET/CT and TB in cervical cancer. Therefore, we hypothesized that TB may be associated with higher metabolic parameters in ${ }^{18}$ F-FDG $\mathrm{PET} / \mathrm{CT}$ because of the aggressive behavior of TB and that the radiomic finding may differ according to TB status.

This study compared the radiomic features on ${ }^{18} \mathrm{~F}-\mathrm{FDG}$ PET/CT and intratumoral heterogeneity according to TB status to develop a prediction model for the TB status using radiomic features of ${ }^{18} \mathrm{~F}-\mathrm{FDG} \mathrm{PET} / \mathrm{CT}$ in patients with cervical cancer.

\section{Materials and Methods}

\subsection{Patients}

Following approval from the Institutional Review Board of Kyungpook National University Chilgok Hospital (KNUCH 2020-03-011), we reviewed the archival medical 
records and hematoxylin and eosin (H\&E)-stained slides of patients with early-stage and locally advanced cervical cancer. The need for informed consent was waived due to the retrospective nature of the study. Between March 2011 and July 2015, a total of 136 patients who underwent radical hysterectomy with pelvic and paraaortic lymphadenectomy for treating early-stage and locally advanced cervical cancer were included. Among the 136 patients, 76 patients underwent preoperative ${ }^{18} \mathrm{~F}$-FDG PET/CT and were enrolled in this study. The enrolled patients were semirandomly divided into a training dataset (51 patients) and a test set (25 patients) using the "doBy" $R$ package while preserving the distribution of ITB status. Patients with a history of preoperative chemotherapy, radiotherapy, or synchronous malignancies were excluded. The patients were clinically staged according to the 2009 International FIGO staging system [18].

\subsection{Histopathological Evaluation}

Specimens were examined from multiple sections of the whole tumor areas and stained with H\&E. For each case, all available specimens were independently reviewed for the detailed histopathological features and the quantitative assessment of TB by two pathologists (J.Y.P and J.Y.P) in a blinded manner, with no knowledge of the clinicopathological data and outcomes.

The pathological parameters included tumor size, FIGO stage, histological subtype, deep stromal invasion, LVI, parametrial invasion, lymph node metastasis, and the number and distribution of TB. TB was defined as an isolated single cancer cell or small cell clusters composed of $\leq 4$ tumor cells located at the advancing edge (PTB) and within the tumor area (ITB).

\section{3. ${ }^{18}$ F-FDG PET/CT Image Acquisition}

All patients fasted for at least $6 \mathrm{~h}$, and their blood glucose levels were determined before the administration of ${ }^{18} \mathrm{~F}-\mathrm{FDG}$. Patients with blood glucose levels $>150 \mathrm{mg} / \mathrm{dL}$ were rescheduled for a later examination, and treatment was administered to maintain a blood glucose concentration $<150 \mathrm{mg} / \mathrm{dL}$ in all participants. Patients received intravenous injections of approximately 5.2 MBq of FDG per kg of body weight and were advised to rest for $1 \mathrm{~h}$ before undergoing ${ }^{18} \mathrm{~F}$-FDG PET/CT imaging. The ${ }^{18} \mathrm{~F}$-FDG PET/CT scans were performed using a Discovery 600 (GE Healthcare, Chicago, IL, USA). Before the PET scan, for attenuation correction, a low-dose CT scan was obtained without contrast enhancement from the skull base to the thigh while the patient was in the supine position and breathing quietly. PET scans were also obtained from the skull base to the thigh at $2.5 \mathrm{~min}$ per bed position. PET images were reconstructed using a $128 \times 128$ matrix and an ordered-subset expectation maximum iterative reconstruction algorithm.

\subsection{Image Interpretation and PET Image Analysis}

The ${ }^{18}$ F-FDG PET/CT images were interpreted by two experienced nuclear medicine physicians (S.Y.J and S.W.L), and a final consensus was achieved for all patients. A positive finding in the uterine cervix was defined as any focus with increased FDG uptake compared to the surrounding normal tissue. Foci of FDG uptake mimicking positive findings in the pelvis, such as urinary activity or a functional ovarian cyst, were excluded from the analysis.

All image analyses were performed using the Advantage Workstation 4.5 software (GE Medical Systems, Waukesha, WI, USA). The primary tumor lesion was delineated by the volume of interest by using an isocontour threshold method based on the SUV, and metabolic PET parameters were assessed. SUVmax values were based on body weight and were calculated using the following formula: $\mathrm{SUVmax}=$ maximum activity in the region of interest (ROI) $(\mathrm{MBq} / \mathrm{g}) /($ injected dose $(\mathrm{MBq}) /$ bodyweight $(\mathrm{g})$ ). SUVmax was designated as the highest value of SUVmax of the primary tumor. The MTV was determined as the volume of voxels with an SUV threshold of the mediastinal blood pool because the mediastinal blood pool is regarded as the preferred site for measuring background activity [19]. The mean SUV of the mediastinal SUV values was determined by drawing an 
ROI over contiguous slices on the descending aorta, carefully excluding the walls from the ROI. The mean SUV of the mediastinal background plus 2 SDs was used as the threshold to automatically calculate the MTV [19]. The TLG was calculated as the MTV multiplied by SUVmean of the lesion. The MTV and TLG were also obtained for the primary tumor.

\subsection{Statistical Analysis}

The differences between subsets were evaluated with a Student's $t$-test or MannWhitney test, and differences between proportions were compared with the chi-square test or Fisher's exact test. Receiver operating characteristic (ROC) curve analysis was performed to identify an optimal cutoff value of metabolic parameters and radiomic finding of ${ }^{18} \mathrm{~F}$ PET/CT for predicting the ITB status. Multiple logistic regression analysis was used to evaluate the metabolic parameters and radiomic findings of ${ }^{18} \mathrm{~F}-\mathrm{PET} / \mathrm{CT}$ for ITB status. The estimated odds ratios (ORs), with 95\% confidence intervals (95\% CIs), are presented. All statistical tests were two-sided, and $p<0.05$ was considered significant. Statistical analysis was performed using SPSS software version 22.0 (SPSS, Chicago, IL, USA), Medcalc version 15.4 (Medcalc Software, Ostend, Belgium), and R version 3.6.3 (R Foundation for Statistical Computing, Vienna, Austria). The R packages "caret", "glmnet", "MASS", and "pROC" were used for analysis.

\subsection{Radiomic Analysis}

Radiomic features were extracted using the LIFEx package (http:/ / www.lifexsoft.org, accessed in 1 July-30 September 2020) [20]. LIFEx was set up using the following input parameters for calculating the features: $64 \mathrm{~Gy}$ levels to resample the ROI content, which was performed in absolute terms between a minimum of 0 and a maximum of 20 [21]. A total of 59 features were extracted from the analysis of the volumes inspected; these indices included conventional parameters, shape and size features, histogram-based features, and second- and high-order-based features. The correction for the partial volume effect was not applied. The analysis included all primary tumor lesions, irrespective of their volume; however, LIFEx calculates the shape and size indices as well as the second-order(gray-level co-occurrence matrix (GLCM)) and high-order-based (neighborhood gray-level different matrix (NGLDM), gray-level run-length matrix, (GLRLM) and gray-level zonelength matrix (GLZLM)) features only for an ROI of at least 64 voxels due to technical reasons. The features calculated are summarized in Table S1.

Each feature value was normalized using $\mathrm{z}$-score normalization $(\mathrm{z}=[\mathrm{x}-$ mean $\{\mathrm{x}\} / \mathrm{SD}$ $\{x\}]$ [standard deviation $\{S D\}])$. The feature selection process consisted of two steps in the training dataset. First, a $t$-test was performed to screen potential features throughout radiomic features and conventional metabolic parameters. Only features with $p<0.05$ were considered significant and entered into the next selection step. Least absolute shrinkage and selection operator (Lasso) regression was used to select key features to build a prediction model, with three-fold cross-validation.

Following feature selection, the prediction models were constructed using a random forest (RF), a support vector machine (SVM), and a neural network (NN) using the training dataset. We built prediction models using only conventional metabolic parameters and both conventional metabolic parameters and radiomic features. The constructed model performance was validated independently in the test dataset by the area under the ROC curve. The improvement of prediction accuracy was assessed with the net reclassification improvement (NRI) and integrated discrimination improvement (IDI) statistics. The R packages "randomForest", "kernlab", "neuralnet", and "PredictABEL" were used to build and evaluate the prediction model.

\section{Results}

\subsection{Clinicopathologic Features and Treatment Outcomes}

The clinicopathologic characteristics of the study participants are listed in Table 1. The predominant FIGO stage was IB1 $(n=43(56.6 \%))$, followed by IB2 $(n=14[18.4 \%))$, 
IIB $(n=10(13.2 \%))$, and IIA $(n=9(11.8 \%))$. The histologic cervical cancer types were as follows: squamous cell carcinoma $(n=91(66.9 \%))$, adenocarcinoma $(n=37(27.2 \%))$, and adenosquamous carcinoma $(n=8(5.9 \%))$ (Table 1$)$. The median ITB count was 3.5 (range, 0-40), and the median PTB count was 4 (range, 0-44). ITB and PTB were observed in $47(61.8 \%)$ and 62 patients $(81.6 \%)$, respectively.

Table 1. Clinicopathological and tumor budding characteristics.

\begin{tabular}{|c|c|}
\hline Variables & $N$ (Range) \\
\hline Age (years) & \\
\hline Mean \pm SD & $47.95 \pm 10.73$ \\
\hline Median (range) & $49(25-74)$ \\
\hline \multicolumn{2}{|l|}{ FIGO stage $(n, \%)$} \\
\hline IB1 & $43,56.6$ \\
\hline IB2 & $14,18.4$ \\
\hline IIA & $9,11.9$ \\
\hline IIB & $10,13.2$ \\
\hline \multicolumn{2}{|l|}{ Histology $(n, \%)$} \\
\hline Squamous cell carcinoma & $54,71.1$ \\
\hline Adenocarcinoma/adenosquamous carcinoma & $22,28.9$ \\
\hline \multicolumn{2}{|l|}{ Tumor size $(\mathrm{cm})$} \\
\hline Mean \pm SD & $3.01 \pm 1.67$ \\
\hline Median (range) & $3(0-8.5)$ \\
\hline Lymphovascular invasion $(n, \%)$ & $59,77.6$ \\
\hline Deep stromal invasion $(n, \%)$ & $46,60.5$ \\
\hline Parametrial invasion $(n, \%)$ & $28,36.8$ \\
\hline Lymph node metastasis $(n, \%)$ & $22,28.9$ \\
\hline \multicolumn{2}{|l|}{ Tumor budding characteristics } \\
\hline \multicolumn{2}{|l|}{ Intratumor budding counts } \\
\hline Mean \pm SD & $6.40 \pm 9.61$ \\
\hline Median (range) & $3.5(0-40)$ \\
\hline \multicolumn{2}{|l|}{ Peritumoral budding counts } \\
\hline Mean \pm SD & $7.49 \pm 9.42$ \\
\hline Median (range) & $4(0-44)$ \\
\hline Intratumoral budding $(n, \%)$ & $47,61.8$ \\
\hline Peritumoral budding $(n, \%)$ & $62,81.6$ \\
\hline
\end{tabular}

FIGO = International Federation of Gynecologic Obstetrics.

\subsection{Comparison of Metabolic Parameters and Radiomic Features of ${ }^{18}$ F-PET/CT According to TB Status}

The median SUVmax was significantly higher in the positive ITB group than in the negative ITB group (11.35 vs. 8.37, $p=0.0406$; Figure 1). However, the median SUVmax did not change significantly according to the PTB status. Among the radiomic features, entropyGLCM (GLCM; $p=0.0111)$, coarseness (NGLDM; $p=0.0497)$, low gray-level run emphasis/long-run low gray-level emphasis (GLRLM; $p=0.0189$ and $p=0.0101$, respectively), low gray-level zone emphasis/short-zone low gray-level emphasis/zonelength nonuniformity zone (GLZLM; $p=0.0137, p=0.0154$, and $p=0.0056$, respectively), sphericity/compacity (shape and size; $p=0.0065$ and $p=0.0108$, respectively), and

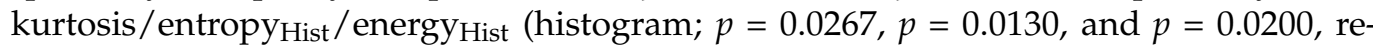
spectively) were significantly different according to the ITB status. However, there were no significantly different radiomic findings according to the PTB status (Table 2). 
(A)
$\begin{array}{lllllllllllll}35 & 32 & 32 & 30 & 32 & 30 & 28 & 20 & 14 & 9 & 6 & 5 & 0\end{array}$

(B)

$\begin{array}{lllll}31 & 32 & 26 & 11 & 0\end{array}$
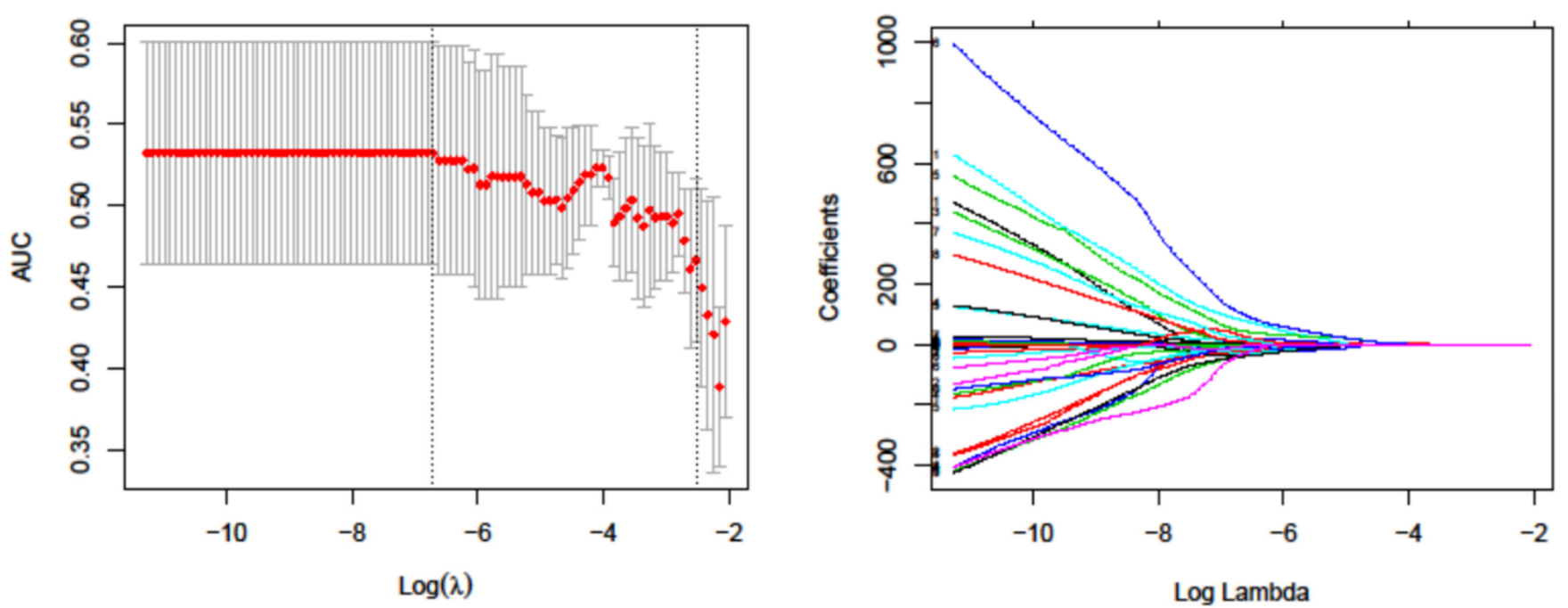

Figure 1. F-18 fluorodeoxyglucose positron emission tomography/computed tomography radiomic feature selection performed by the least absolute shrinkage and selection operator (Lasso) regularization method. (A) Area under the curve was drawn versus $\log (\lambda)$ by the 5 -fold cross-validation. The vertical dotted line defines the optimal $\lambda$ value. The optimal $\lambda$ of 0.0012 , with $\log (\lambda)$ of -6.7061 was selected; (B) Lasso coefficient profiles of the 48 potential PET features as selected by the $t$-test. Twenty-seven features were selected with the optimal $\lambda$.

\subsection{Multiple Logistic Regression Analysis for ITB Status}

Univariate and multivariate analyses were performed to evaluate the correlation between ${ }^{18} \mathrm{~F}$-FDG PET/CT values and the ITB status (Table 3). Among the significant parameters of conventional metabolic parameters (SUVmax, MTV, and TLG) and each radiomic finding (GLCM, NGLDM, GLZLM, shape and size, and histogram) in univariate analysis, the most significant parameters (the lowest $p$ value) were included in multivariate analysis for the inhibition of conflicting each parameter. In univariate analysis, SUVmax (OR, 3.34; 95\% CI, 1.27-8.79; $p=0.0146)$, TLG (OR, 4.42\%; 95\% CI, 1.33-14.72; $p=0.0154)$, entropyGLCM (OR, 5.36; 95\% CI, 1.95-14.69; $p=0.0011)$, coarseness (OR, 3.94; 95\% CI, $1.41-11.03 ; p=0.0090)$, low gray-level run emphasis $(\mathrm{OR}, 3.45 ; 95 \% \mathrm{CI}, 1.26-9.47 ; p=0.0161)$, long-run low gray-level emphasis (OR, 3.34; 95\% CI, 1.27-8.79; $p=0.0146)$, low graylevel zone emphasis (OR, 4.05; 95\% CI, 1.52-10.82; $p=0.0052)$ short-zone low gray-level emphasis (OR, 3.94; 95\% CI, 1.41-11.03; $p=0.0090)$, zone-length nonuniformity zone (OR, 9.16; 95\% CI, 1.27-8.79; $p=0.0146)$, sphericity (OR, 6.09; 95\% CI, 2.15-17.28; $p=0.0007)$, compacity (OR, 8.73; 95\% CI, 2.48-30.76; $p=0.0007)$, kurtosis (OR, 3.96; 95\% CI, 1.38-11.38; $p=0.0106)$, Entropy ${ }_{\text {Hist }}(\mathrm{OR}, 5.98 ; 95 \% \mathrm{CI}, 2.12-16.86 ; p=0.0007)$, and Energy Hist $_{\text {(OR, }}$ (.98; 95\% CI, 2.12-16.86; $p=0.0007$ ) were significant parameters that correlated with positive ITB. Multivariate analysis with the entered methods showed that only compacity (OR, 5.00; 95\% CI, 1.16-21.53; $p=0.0305)$ remained an independent parameter correlated with positive ITB (Table 3). 
Table 2. Comparison of radiomic features according to the tumor budding status.

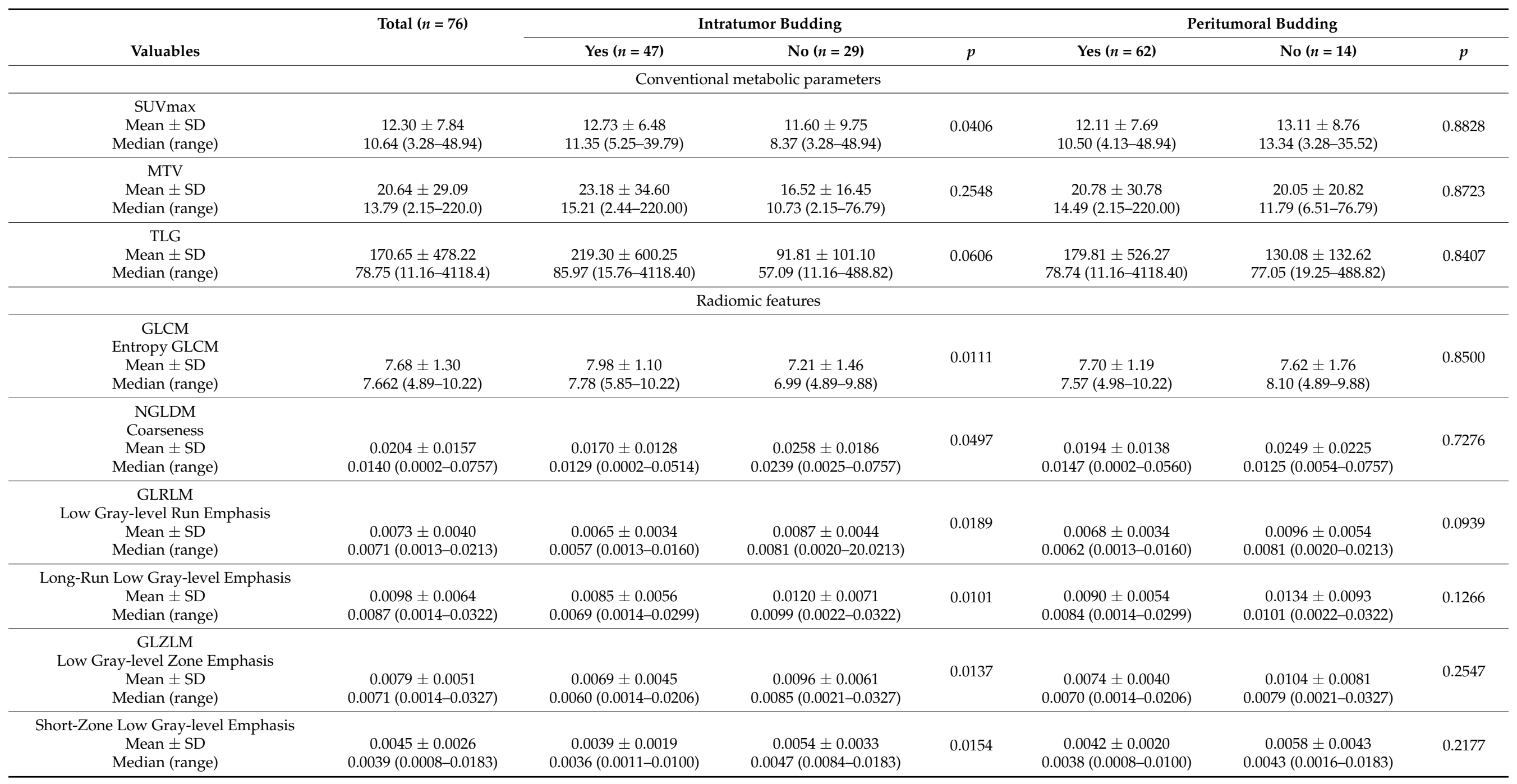


Table 2. Cont.

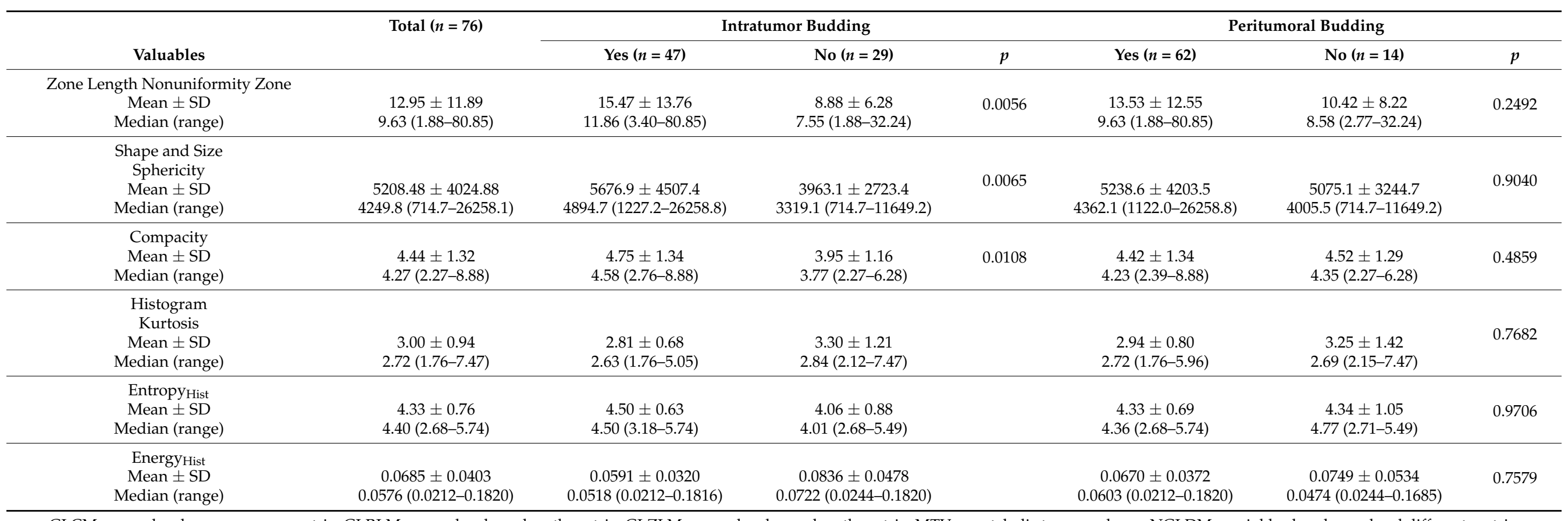

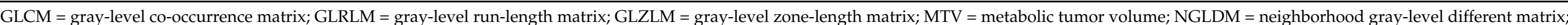

SUVmax = maximum standardized uptake; TLG = total lesion glycolysis. 
Table 3. Multiple logistic regression analysis for the evaluation of the correlation between radiomic features and intratumor budding status.

\begin{tabular}{|c|c|c|c|c|c|c|}
\hline \multirow[b]{2}{*}{ Variables } & \multicolumn{3}{|c|}{ Univariate Analysis } & \multicolumn{3}{|c|}{ Multivariate Analysis } \\
\hline & Odds Ratio & $95 \%$ CI & $p$ & Odds Ratio & $95 \%$ CI & $p$ \\
\hline \multicolumn{7}{|c|}{ Conventional metabolic parameters } \\
\hline SUVmax (>8.858) & 3.3393 & $1.2685-8.7908$ & 0.0146 & 2.4973 & $0.8933-6.9810$ & 0.0810 \\
\hline $\operatorname{MTV}\left(\mathrm{cm}^{3},>16.3\right)$ & 2.5385 & $0.9092-7.0869$ & 0.0753 & & & \\
\hline TLG $(>32.0247)$ & 4.4211 & $1.3282-14.7159$ & 0.0154 & & & \\
\hline \multicolumn{7}{|c|}{ Radiomic features } \\
\hline GLCM & & & & & & \\
\hline EntropyGLCM (>7.1782) & 5.3554 & $1.9520-14.6928$ & 0.0011 & 0.6139 & $0.0708-5.3208$ & 0.6579 \\
\hline NGLDM & & & & & & \\
\hline Coarseness $(\leq 0.0254)$ & 3.9407 & $1.4085-11.0251$ & 0.0090 & 1.0604 & $0.2057-5.4660$ & 0.9441 \\
\hline GLRLM & & & & & & \\
\hline $\begin{array}{l}\text { Low Gray-level Run Emphasis } \\
\qquad(\leq 0.0081)\end{array}$ & 3.4533 & $1.2588-9.4739$ & 0.0161 & & & \\
\hline $\begin{array}{l}\text { Long-Run Low Gray-level } \\
\text { Emphasis }(\leq 0.0094)\end{array}$ & 3.3393 & $1.2685-8.7908$ & 0.0146 & 1.6936 & $0.4822-5.9481$ & 0.4111 \\
\hline \multicolumn{7}{|l|}{ GLZLM } \\
\hline $\begin{array}{l}\text { Low Gray-level Zone } \\
\text { Emphasis }(\leq 0.0074)\end{array}$ & 4.0533 & $1.5196-10.8155$ & 0.0052 & & & \\
\hline $\begin{array}{c}\text { Short-Zone Low Gray-level } \\
\text { Emphasis }(\leq 0.005)\end{array}$ & 3.9407 & $1.4085-11.0251$ & 0.0090 & & & \\
\hline $\begin{array}{l}\text { Zone Length Nonuniformity } \\
\text { Zone }(>14.1652)\end{array}$ & 9.1607 & $1.9444-43.1600$ & 0.0051 & 5.3971 & $0.9464-30.7790$ & 0.0577 \\
\hline \multicolumn{7}{|l|}{ Shape and Size } \\
\hline Sphericity (>4160.0834) & 6.0893 & $2.1463-17.2763$ & 0.0007 & & & \\
\hline Compacity (>3.4057) & 8.7344 & $2.4798-30.7648$ & 0.0007 & 5.0047 & $1.1636-21.5253$ & 0.0305 \\
\hline \multicolumn{7}{|l|}{ Histogram } \\
\hline Kurtosis $(\leq 3.1264)$ & 3.9609 & $1.3783-11.3826$ & 0.0106 & & & \\
\hline Entropy Hist $_{\text {His }}(>4.0608)$ & 5.9815 & $2.1219-16.8615$ & 0.0007 & & & \\
\hline Energy $_{\text {Hist }}(\leq 0.0688)$ & 5.9815 & $2.1219-16.8615$ & 0.0007 & 2.9011 & $0.4526-18.5937$ & 0.2611 \\
\hline
\end{tabular}

$\mathrm{CI}$ = confidence interval GLCM = gray-level co-occurrence matrix; GLRLM = gray-level run-length matrix; GLZLM = gray-level zone-length matrix; MTV = metabolic tumor volume; NGLDM = neighborhood gray-level different matrix; SUVmax = maximum standardized uptake; $\mathrm{TLG}=$ total lesion glycolysis.

\subsection{Predicting Model for ITB Status Using Radiomic Features of ${ }^{18}$ F-FDG PET/CT}

Forty-eight features were significant parameters in the $t$-test and were subjected to a further selection step by the Lasso regularization (Figure 1). Among them, the final 27 remaining features (SUVmax, MTV, SUV_Skewness, discretized_SUVmax, discretized_SUV_Skewness, discretized_SUV_Kurtosis, discretized_SUVpeak_Sphere, discretized_HISTO_ExcessKurtosis, GLCM_Entropy_log2, GLCM_Dissimilarity, GLRLM_LRE, GLRLM_LGRE, GLRLM_SRHGE, GLRLM_LRLGE, GLRLM_GLNU, GLRLM_RLNU, NGLDM_Contrast, NGLDM_Busyness, GLZLM_SZE, GLZLM_LZE0, GLZLM_LGZE, GLZLM_HGZE, GLZLM_SZLGE, GLZLM_SZHGE, and GLZLM_LZLGE) were selected. Supplementary Figure $\mathrm{S} 1$ shows the ROC curves of the prediction models by three machine learning algorithms in the training and test datasets. The area under the curve (AUC) values of the prediction models constructed by the RF, SVM, and NN were $0.752,0.784$, 0.752 , respectively in the test dataset. Figure 2 shows the ROC curves of the prediction models built using only conventional metabolic parameters and conventional metabolic parameters+radiomics features in the test datasets. The area under the curve (AUC) values of the prediction models using conventional metabolic parameters constructed by the RF, SVM, and NN were $0.673,0.719$, and 0.712 , respectively (Table 4 ). With the addition of radiomic features, AUC values in the test dataset were $0.752,0.784,0.752$ in the RF, SVM, and NN models, respectively. The NRI values for the addition of radiomic features to conventional metabolic parameters were $0.183,0.105$, and 0.275 in RF, SVM, and NN 
models, respectively. This consisted of a respective $18.3 \%, 10.5 \%$, and $27.5 \%$ improvement in classification by adding radiomic parameters into prediction models, although these gains were not statistically significant.

(A)

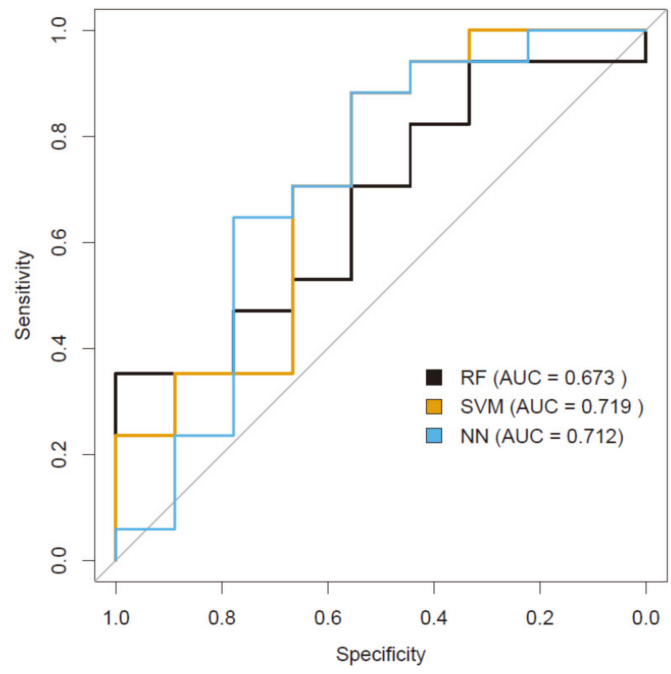

(B)

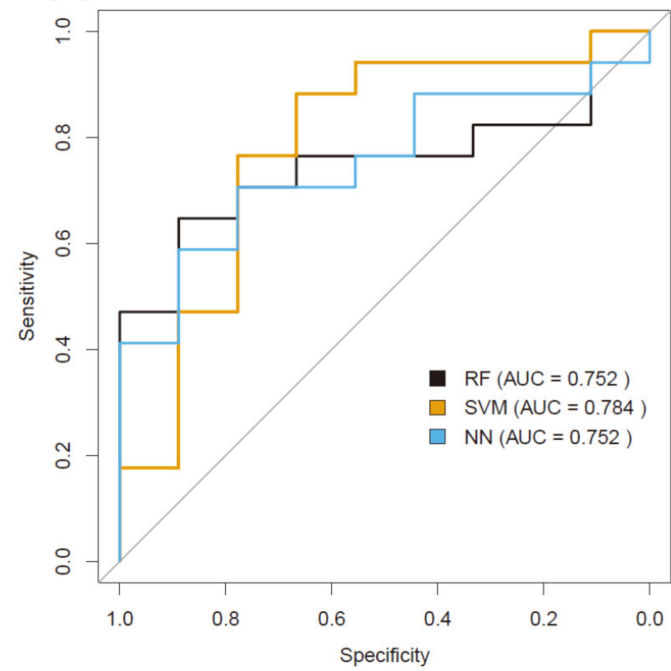

Figure 2. Receiver operating characteristic curves of the prediction models constructed by the random forest, support vector machine, and neural network algorithms using conventional metabolic parameters only (A) and conventional metabolic parameters + radiomic features $(\mathbf{B})$ in the test dataset.

Table 4. Performance metrics of prediction models for predicting tumor budding status in the test dataset.

\begin{tabular}{cccccc}
\hline Model & AUC (95\% CI) & NRI (95\% CI) & $p$ & IDI (95\% CI) & $p$ \\
\hline \multicolumn{7}{c}{ Conventional metabolic parameters } \\
RF & $0.673(0.454-0.893)$ & Reference & - & Reference \\
SVM & $0.719(0.488-0.950)$ & Reference & - & Reference \\
NN & $0.712(0.469-0.956)$ & Reference & - & Reference \\
\hline \multicolumn{7}{c}{ Conventional metabolic parameters + radiomic features } \\
\hline RF & $0.752(0.561-0.943)$ & $0.183(-0.115-0.482)$ & 0.229 & $0.183(-0.129-0.495)$ & - \\
SVM & $0.784(0.576-0.993)$ & $0.105(-0.334-0.543)$ & 0.640 & $0.105(-0.359-0.543)$ & 0.658 \\
NN & $0.752(0.561-0.942)$ & $0.275(-0.158-0.707)$ & 0.214 & $0.275(-0.178-0.727)$ & 0.234 \\
\hline
\end{tabular}

AUC $=$ area under the curve; $\mathrm{CI}=$ confidence interval; $\mathrm{NRI}=$ net reclassification improvement; $\mathrm{IDI}=$ integrated discrimination improvement; $\mathrm{RF}=$ random forest; $\mathrm{SVM}=$ support vector machine; $\mathrm{NN}=$ neural network.

\section{Discussion}

In this study, the radiomic features of ${ }^{18} \mathrm{~F}-\mathrm{FDG}$ PET/CT were associated with TB status, especially in the ITB status. Among the 59 features, 12 features were significantly different according to the ITB status in univariate logistic regression analysis. Among these 12 features, compacity was the most significant parameter for the ITB status in multivariate logistic regression analysis. Moreover, we developed the prediction model for the ITB status by the 3 commonly used machine learning classifiersusing conventional metabolic parameters and radiomic features, and the mean AUC was 0.763 .

Radiomics is a relatively new and evolving field in medical imaging in which many features are extracted from medical image analysis and interpretation using bioinformatic approaches [15]. Furthermore, radiomics precision medicine and tumor heterogeneity have recently become a hot topic in oncological medicine [22]. At the biological level, it has been recognized that the heterogeneity of the tumor microenvironment might be reflected in medical images, with respect to cellular density, proliferation, angiogenesis, hypoxia, receptor expression, necrosis, fibrosis, and inflammation, all of which may contribute to a more aggressive phenotype and poor treatment responses [23]. Therefore, the radiomic 
signature may represent a segmentation of tumor subregions with different biological characteristics and contribute to treatment response and prognosis.

In colorectal cancer, high ITB has been shown to correlate with higher tumor grade, higher $\mathrm{pT}$ stage, lymphatic invasion, vascular invasion, nodal metastasis, and shorter survival time [24]. Moreover, ITB may lead to more aggressive clinicopathologic characteristics through a similar mechanism to that of PTB, such as increased extracellular matrix degradation, increased migration, and loss of cell adhesion [16]. Therefore, we hypothesized that ITB may be associated with higher metabolic parameters due to its aggressive nature.

To date, only one study has demonstrated a correlation between tumor cancer cell metabolism and the morphological features of aggressiveness as assessed by microscopy such as TB [24]. In an earlier study, the MTV was higher in the TB group than in the nonTB group, with marginal significance $(p=0.06)$ in laryngeal and pharyngeal carcinoma [25]. In this study, SUVmax was significantly different according to the ITB status $(p=0.0406)$. Moreover, SUVmax and TLG were associated with the ITB status in univariate logistic regression analysis ( $p=0.0146$ and $p=0.0154$, respectively). The aggressive nature of ITB may present as higher metabolic parameters in ${ }^{18} \mathrm{~F}-\mathrm{FDG}$ PET/CT.

EMT of primary tumor tissues may lead to the loss of cell-to-cell adhesion and occurrence ITB. Consequently, ITB may lead to the segmentation of tumor subregions with different biological characteristics and may contribute to tumor heterogeneity. However, to our knowledge, no study has reported on the correlation between ITB and radiomic findings. In this study, 12 features were associated with the ITB status and compacity was a powerful biomarker representing ITB status in multivariate logistic regression analysis. Previous studies demonstrated that among the radiomic features, compacity was the most significant covariate to predict local control and survival in hepatocellular carcinoma [26].

The results of our previous study showed that tumors with high TB were significantly associated with LVI, deep stromal invasion, parametrial invasion, and lymph node metastasis in cervical cancer [6]. Preoperative prediction of the TB status may help in the development of personalized medicine, such as decisions on the radicality of surgery or the extent of lymphadenectomy. However, TB status was finally determined by postoperative surgical specimens. To date, there is no modality that can estimate TB status preoperatively. Therefore, prediction models were constructed for ITB status using both conventional metabolic parameters and radiomic features from ${ }^{18} \mathrm{~F}-\mathrm{FDG}$ PET/CT scans. Among the 48 features, which were significant parameters in the univariate logistic regression, 27 features were selected by the $t$-test and Lasso regularization. The AUC values of the prediction models were $>0.75$ in the test dataset. The prediction performance was improved in all classifiers, although they were not statistically significant. One possible reason for statistical insignificance is that the number of test dataset was too small $(n=25)$ and may underestimate the statistical power.

The main limitations of this study are its retrospective nature, heterogeneity of the sample, and small sample size, which may have contributed to selection bias. Additionally, as this is a single-center study, the generalization of our findings is limited. Despite these limitations, our study offers some unique and significant findings, in that we showed correlations between radiomic findings and the TB status for the first time and established the prediction models for the ITB status using radiomic features in ${ }^{18} \mathrm{~F}-\mathrm{FDG}$ PET/CT.

In conclusion, higher metabolic quantities were observed in the positive ITB group than in the negative ITB group. Radiomic findings in ${ }^{18} \mathrm{~F}-\mathrm{FDG}$ PET/CT were associated with ITB status, and among these features, compacity was the most significant covariate for the ITB status. Furthermore, prediction models for ITB status using radiomic findings in ${ }^{18} \mathrm{~F}$-FDG PET/CT may contribute to personalized medicine in cervical cancer.

Supplementary Materials: The following are available online at https:/ / www.mdpi.com/article/10 .3390/diagnostics11081517/s1, Figure S1. Receiver operating characteristic curves of the prediction models constructed by the random forest, support vector machine, and neural network algorithms using metabolic and radiomic features in the training dataset (A) and test dataset (B). Table S1: Radiomic features calculated from 18F-FDG PET/CT images in Lifex. 
Author Contributions: Conceptualization: G.O.C., S.Y.J., S.-H.P., J.Y.P., and H.S.H.; Methodology: G.O.C., S.J.K., Y.H.L., N.J.-Y.P., J.Y.P., S.Y.J., and S.-W.L.; Formal analysis and investigation: G.O.C., S.H.P., S.Y.J., Y.H.L., S.-W.L., and D.G.H.; Writing-original draft preparation: G.O.C., S.-H.P., N.J.-Y.P., S.Y.J., and S.J.K.; Writing-review and editing: Y.H.L., N.J.-Y.P., J.Y.P., S.-W.L., D.G.H., and H.S.H.; Funding acquisition: G.O.C., S.-W.L., and H.S.H.; Resources: G.O.C., S.Y.J., S.-H.P., S.J.K., N.J.-Y.P., Y.H.L., and H.S.H.; Supervision: S.-W.L., D.G.H., J.Y.P., and H.S.H. All authors have read and agreed to the published version of the manuscript.

Funding: This work was supported by the National Research Foundation of Korea (NRF) grant funded by the Korean government (MIST) (No. 2020R1G1A1102848), and a grant from the Korea Health Technology R\&D Project through the Korea Health Industry Development Institute (KHIDI), funded by the Ministry of Health \& Welfare, Republic of Korea (Grant No. HI16C1501).

Institutional Review Board Statement: The study was conducted according to the guidelines of the Declaration of Helsinki, and approved by the Institutional Review Board of Kyungpook National University Chilgok Hospital (IRB No. KNUCH 2020-03-011).

Informed Consent Statement: Patient consent was waived due to the retrospective nature of the study.

Data Availability Statement: The data presented in this study are available on request from the corresponding author. The data are not publicly available due to privacy and ethical restrictions.

Conflicts of Interest: The authors declare no conflict of interest.

\section{References}

1. Lugli, A.; Kirsch, R.; Ajioka, Y.; Bosman, F.; Cathomas, G.; Dawson, H.; Zimaity, H.E.; Fléjou, J.-F.; Hansen, T.P.; Hartmann, A.; et al. Recommendations for reporting tumor budding in colorectal cancer based on the International Tumor Budding Consensus Conference (ITBCC) 2016. Mod. Pathol. 2017, 30, 1299-1311. [CrossRef]

2. Graham, R.P.; Vierkant, R.A.; Tillmans, L.S.; Wang, A.H.; Laird, P.W.; Weisenberger, D.J.; Lynch, C.F.; French, A.J.; Slager, S.L.; Raissian, Y.; et al. Tumor Budding in Colorectal Carcinoma: Confirmation of Prognostic Significance and Histologic Cutoff in a Population-based Cohort. Am. J. Surg. Pathol. 2015, 39, 1340-1346. [CrossRef] [PubMed]

3. Almangush, A.; Karhunen, M.; Hautaniemi, S.; Salo, T.; Leivo, I. Prognostic value of tumour budding in oesophageal cancer: A meta-analysis. Histopathology 2016, 68, 173-182. [CrossRef]

4. Almangush, A.; Salo, T.; Hagström, J.; Leivo, I. Tumour budding in head and neck squamous cell carcinoma-A systematic review. Histopathology 2014, 65, 587-594. [CrossRef]

5. Park, J.Y.; Hong, D.G.; Chong, G.O.; Park, J.Y. Tumor Budding is a Valuable Diagnostic Parameter in Prediction of Disease Progression of Endometrial Endometrioid Carcinoma. Pathol. Oncol. Res. 2019, 25, 723-730. [CrossRef]

6. Park, J.Y.; Chong, G.O.; Park, J.Y.; Chung, D.; Lee, Y.H.; Lee, H.J.; Hong, D.G.; Han, H.S.; Lee, Y.S. Tumor budding in cervical cancer as a prognostic factor and its possible role as an additional intermediate-risk factor. Gynecol. Oncol. 2020, 159, 157-163. [CrossRef]

7. Wong, T.Z.; Jones, E.L.; Coleman, R.E. Positron emission tomography with 2-deoxy-2-[(18)F]fluoro-D-glucose for evaluating local and distant disease in patients with cervical cancer. Mol. Imaging Biol. 2004, 6, 55-62. [CrossRef]

8. Han, L.; Wang, Q.; Zhao, L.; Feng, X.; Wang, Y.; Zou, Y.; Li, Q. A Systematic Review and Meta-Analysis of the Prognostic Impact of Pretreatment Fluorodeoxyglucose Positron Emission Tomography/Computed Tomography Parameters in Patients with Locally Advanced Cervical Cancer Treated with Concomitant Chemoradiotherapy. Diagnostics 2021, 11, 1258. [CrossRef]

9. Kim, D.H.; Jung, J.H.; Son, S.H.; Kim, C.Y.; Hong, C.M.; Oh, J.R.; Jeong, S.Y.; Lee, S.W.; Lee, J.; Ahn, B.C. Prognostic Significance of Intratumoral Metabolic Heterogeneity on 18F-FDG PET/CT in Pathological N0 Non-Small Cell Lung Cancer. Eur. J. Nucl. Med. Mol. Imaging 2014, 41, 2051-2057. [CrossRef]

10. Tixier, F.; Le Rest, C.C.; Hatt, M.; Albarghach, N.; Pradier, O.; Metges, J.P.; Corcos, L.; Visvikis, D. Intratumor heterogeneity characterized by textural features on baseline 18F-FDG PET images predicts response to concomitant radiochemotherapy in esophageal cancer. J. Nucl. Med. 2011, 52, 369-378. [CrossRef]

11. Cheng, N.M.; Fang, Y.H.; Chang, J.T.; Huang, C.G.; Tsan, D.L.; Ng, S.H.; Wang, H.M.; Lin, C.Y.; Liao, C.T.; Yen, T.C. Textural features of pretreatment 18F-FDG PET/CT images: Prognostic significance in patients with advanced T-stage oropharyngeal squamous cell carcinoma. J. Nucl. Med. 2013, 54, 1703-1709. [CrossRef]

12. Chong, G.O.; Lee, W.K.; Jeong, S.Y.; Park, S.H.; Lee, Y.H.; Lee, S.W.; Hong, D.G.; Kim, J.C.; Lee, Y.S. Prognostic value of intratumoral metabolic heterogeneity on F-18 fluorodeoxyglucose positron emission tomography/computed tomography in locally advanced cervical cancer patients treated with concurrent chemoradiotherapy. Oncotarget 2017, 8, 90402-90412. [CrossRef]

13. Reuzé, S.; Orlhac, F.; Chargari, C.; Nioche, C.; Limkin, E.; Riet, F.; Escande, A.; Haie-Meder, C.; Dercle, L.; Gouy, S.; et al. Prediction of cervical cancer recurrence using textural features extracted from 18F-FDG PET images acquired with different scanners. Oncotarget 2017, 27, 43169-43179. [CrossRef] 
14. Li, X.R.; Jin, J.J.; Yu, Y.; Wang, X.H.; Guo, Y.; Sun, H.Z. PET-CT radiomics by integrating primary tumor and peritumoral areas predicts E-cadherin expression and correlates with pelvic lymph node metastasis in early-stage cervical cancer. Eur. Radiol. 2021, 31, 5967-5979. [CrossRef]

15. Cook, G.J.R.; Azad, G.; Owczarczyk, K.; Siddique, M.; Goh, V. Challenges and Promises of PET Radiomics. Eur. J. Nucl. Med. Mol. Imaging 2013, 40, 133-140. [CrossRef]

16. Zlobec, I.; Lugli, A. Epithelial mesenchymal transition and tumor budding in aggressive colorectal cancer: Tumor budding as oncotarget. Oncotarget 2010, 1, 651-661. [CrossRef] [PubMed]

17. Thiery, J.P.; Sleeman, J.P. Complex networks orchestrate epithelial-mesenchymal transitions. Nat. Rev. Mol. Cell Biol. 2006, 7, 131-142. [CrossRef] [PubMed]

18. Pecorelli, S. Revised FIGO staging for carcinoma of the vulva, cervix, and endometrium. Int. J. Gynaecol. Obstet. 2009, 105, 103-104. [CrossRef]

19. Ziai, P.; Hayeri, M.R.; Salei, A.; Salavati, A.; Houshmand, S.; Alavi, A.; Teytelboym, O.M. Role of Optimal Quantification of FDG PET Imaging in the Clinical Practice of Radiology. Radiographics 2016, 36, 481-496. [CrossRef]

20. Wahl, R.L.; Jacene, H.; Kasamon, Y.; Lodge, M.A. From RECIST to PERCIST: Evolving Considerations for PET response criteria in solid tumors. J. Nucl. Med. 2009, 50 (Suppl. 1), 122S-150S. [CrossRef]

21. Nioche, C.; Orlhac, F.; Boughdad, S.; Reuzé, S.; Goya-Outi, J.; Robert, C.; Pellot-Barakat, C.; Soussan, M.; Frouin, F.; Buvat, I. LIFEx: A Freeware for Radiomic Feature Calculation in Multimodality Imaging to Accelerate Advances in the Characterization of Tumor Heterogeneity. Cancer Res. 2018, 78, 4786-4789. [CrossRef]

22. Valdora, F.; Houssami, N.; Rossi, F.; Calabrese, M.; Tagliafico, A.S. Rapid review: Radiomics and breast cancer. Breast Cancer Res. Treat. 2018, 169, 217-229. [CrossRef]

23. Junttila, M.R.; de Sauvage, F.J. Influence of tumour micro-environment heterogeneity on therapeutic response. Nature 2013, 501, 346-354. [CrossRef]

24. Lugli, A.; Vlajnic, T.; Giger, O.; Karamitopoulou, E.; Patsouris, E.S.; Peros, G.; Terracciano, L.M.; Zlobec, I. Intratumoral budding as a potential parameter of tumor progression in mismatch repair-proficient and mismatch repair-deficient colorectal cancer patients. Hum. Pathol. 2011, 42, 1833-1840. [CrossRef]

25. Karpathiou, G.; Gavid, M.; Prevot-Bitot, N.; Dhomps, A.; Dumollard, J.M.; Vieville, M.; Lelonge, Y.; Prades, J.M.; Froudarakis, M.; Peoc'h, M. Correlation between Semiquantitative Metabolic Parameters after PET/CT and Histologic Prognostic Factors in Laryngeal and Pharyngeal Carcinoma. Head Neck Pathol. 2020, 14, 724-732. [CrossRef]

26. Cozzi, L.; Dinapoli, N.; Fogliata, A.; Hsu, W.C.; Reggiori, G.; Lobefalo, F.; Kirienko, M.; Sollini, M.; Franceschini, D.; Comito, T.; et al. Radiomics based analysis to predict local control and survival in hepatocellular carcinoma patients treated with volumetric modulated arc therapy. BMC Cancer 2017, 17, 829. [CrossRef] 